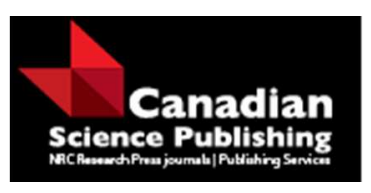

Canadian Journal of Physics

Revue canadienne de physique

\title{
Numerical simulations of heat transfer to a third grade fluid flowing between two parallel plates
}

\begin{tabular}{|r|l|}
\hline Journal: & Canadian Journal of Physics \\
\hline Manuscript ID & cjp-2017-0113.R4 \\
\hline Manuscript Type: & Article \\
\hline Date Submitted by the Author: & 08-Aug-2017 \\
\hline Complete List of Authors: & $\begin{array}{l}\text { Rasheed, Amer; Lahore University of Management Sciences } \\
\text { Ali, Fariha; COMSATS Institute of Information Technology - Wah Campus } \\
\text { Kamran, M.; COMSATS Inst Informat Technol, Mathematics } \\
\text { Akbar, Tanvir; COMSATS Institute of Information Technology, Mathematics } \\
\text { Khan, Sohail; COMSATS Institute of Information Technology - Wah } \\
\text { Campus, Mathematics }\end{array}$ \\
\hline Keyword: & $\begin{array}{l}\text { Heat transfer, Third grade fluid, Non-Newtonian fluid, Lagrange-Galerkin } \\
\text { method, Finite element method }\end{array}$ \\
\hline $\begin{array}{r}\text { Is the invited manuscript for } \\
\text { consideration in a Special } \\
\text { Issue? : }\end{array}$ & N/A \\
\hline
\end{tabular}




\title{
Numerical simulations of heat transfer to a third grade fluid flowing between two parallel plates
}

\author{
Amer Rasheed ${ }^{1}$, Fariha $\mathrm{Ali}^{2},{ }^{*}$ Muhammad Kamran ${ }^{2}$, Tanvir Akbar ${ }^{3}$, Sohail Ahmad Khan ${ }^{2}$ \\ ${ }^{1}$ Department of Mathematics, School of Science and Engineering, \\ Lahore University of Management Sciences, Lahore, Pakistan \\ ${ }^{2}$ Department of Mathematics, COMSATS Institute of Information Technology, \\ Wah Cantt, Pakistan \\ ${ }^{3}$ Department of Mathematics, COMSATS Institute of Information Technology, \\ Islamabad, Pakistan
}

October 10, 2017

\begin{abstract}
This investigation deals with numerical treatment of heat transfer flow of a third grade fluid between two infinite parallel plates subject to no-slip condition at boundary and no-temperature jump. Three flow configurations, Couette, Poiseuille and plane Couette-Poiseuille have been discussed. Approximate solutions using Lagrange-Galerkin method to Couette, Poiseuille and Couette-Poiseuille flow problems are computed and delineated. It has been substantiated that the fluid rheology and heat transfer phenomenon are greatly influenced by the third grade flow parameters, Brinkman number, and pressure gradient. A rigorous mathematical exposition of the numerical scheme is provided. Since, no à priori assumptions are made on pertinent flow parameters, apart from those due to thermodynamic stability, the results presented in this investigation are also valid for their large values.
\end{abstract}

AMS subject classifications 2000. 76D15, 35C15; 76A05

Key words. Heat transfer; Third grade fluid; Non-Newtonian fluid, Finite element method.

\section{Introduction}

The emergence of non-Newtonian flows in nature, industrial processes and different engineering systems has influenced a great deal of experimental studies and triggered thereby associated intriguing mathematical problems over a few past decades $[1,2,3,4,5,6,7,8,9,10]$. It is now widely apprehended that the Newtonian paradigm, based on a linear stress-strain relationship, is neither always realistic nor accurate in describing the underlying fluid rheology [11].

In certain industrial procedures, where a fluid is subjected to heating, its rheology eloquently varies with respect to temperature. Reference can be made to manufacturing processes for producing parts by injecting a material into a mold, drilling of an oil-well or combustion involving

*Address Correspondence to Muhammad Kamran (getkamran@gmail.com) 
fossil fuel for instance $[12,13,14]$. Bestowed by widespread applications, the understanding of heat transfer phenomena in fluids is therefore a substantive issue that has been dealt with in numerous studies recently.

Couette and planar Poiseuille flow are both steady flows between two infinitely long parallel plates a fixed distance apart. The difference is that in Couette flow one of the plates has a velocity in its own plane (the other plate is at rest) as a result of the application of a shear stress, and there is no pressure gradient in the fluid. In contrast in planar Poiseuille flow both plates are at rest and the flow is caused by a pressure gradient in the direction parallel to the plates. The Couette-Poiseuille flow, as a fundamental problem, has been widely studied both theoretically and experimentally for a long time. For a Newtonian fluid, a linear velocity profile for a Couette flow and a parabolic velocity profile for a Poiseuille flow can be derived with no difficulty. However, signicant variations of these flows emerge when non-Newtonian effects are taken into account. During the last several decades, there were plenty of studies on the Couette-Poiseuille flow with various non-Newtonian fluid models. There are a number of theoretical results concerning Couette-Poiseuille flow [15, 16, 17, 18, 19]

The principle concern of this investigation is the numerical exploration of heat transfer phenomenon in fluids of grade three bounded between two parallel plates under no-slip and no temperature jump assumptions. It is worthy to mention that identical problem has been studied for numerical and asymptotic solutions for different flow and geometric configurations; see for instance $[20,21,22,23,24]$. The heat transfer flow under consideration has been mathematically modeled by Siddiqui et al. in [21] wherein they provided a solution using the paradigm of homotopy perturbation method (HPM) of He. Subsequently, Roohi et al. in [23] used the Liao's homotopy analysis method (HAM) to find an asymptotic solution. Makukula et $a l$. [22] devised a quasi-linearization based spectral homotopy analysis approach. It is valuable to note that the asymptotic solutions are valid for small values of pertinent flow parameters. In fact, in [24] Lipscombe derived the exact solution to the aforementioned problem in parametric form and proved exclusively that the HAM solutions are not valid for large values of flow parameter. Moreover, Sajid et al. in [25] provided a comparison between HPM and HAM wherein it is proved that HAM solution may diverge for strongly non-linear problems.

Due to unfitness of asymptotic approaches for high values of flow parameters, Mahmood et al. [20] attempted to find numerical solution to the heat transfer flow problem using finite element method (FEM). They used a standard Galerkin method with first degree polynomial interpolation to resolve boundary value problems corresponding to different types of flow, precisely, Couette, Poiseuille and Couette-Poiseuille. Unfortunately, the mathematical exploration was neither rigorous nor well adopted to the non-linearity of governing equations. As it will be discussed later on, at least second degree interpolation functions are required to effectively take into account the non-linearity of problem.

The aim of this work is twofold:

1) To provide a rigorous and justified mathematical exploration of the heat transfer flow using a finite element method.

2) To take into account the strong non-linearity of the problem, large values of pertinent parameters and their influence on flow profile in three flow configurations: flow due to the movement of the upper plate (Couette flow), flow due to the pressure gradient (Poiseuille flow) and flow due to both the upper plate movement and the pressure gradient (plane Couette-Poiseuille or the generalized plane Couette flow).

The rest of this article is organized in the following manner. Heat transfer flow problem is presented and mathematically formulated in section 2 . section 3 deals with numerical treatment 
of the flow problem. Finite element method is presented and the numerical scheme to obtain and invert global assembly matrix is provided. Numerical simulations depicting the influence of assorted parameters and error curves are given in section 4 The investigation is finally summarized in section 6. Finally, some explicit calculations are detailed in Appendix A.

\section{Mathematical Formulation}

The principle objective of this section is to present and mathematically formulate the heat transfer flow problem subject to conditions of no-slip at the boundary and no-jump in the temperature. It is widely presumed that the velocity of particles adjacent to the solid boundaries often coincides with the velocity of boundary, that is, fluid does not slip relative to the solid boundary. This effect is commonly known as the condition of no-slip at the boundary. It is important to precise that the boundary value problems associated to different types of flow provided in this section have been first derived by Siddiqui et al. in [21]. However, as these are the building blocks of present study, we briefly elaborate the boundary value problems and provide their non-dimensional forms.

\section{Nomenclature}

\begin{tabular}{|ll|}
\hline $2 L$ & distance between plates \\
$\psi$ & temperature field \\
$\mathbf{U}$ & velocity field \\
$\rho$ & fluid density \\
$\sigma$ & stress tensor \\
$k$ & thermal conductivity of the fluid \\
$\sigma$ & Cauchy stress tensor \\
$t r$ & trace of matrix \\
$p$ & hydro-static pressure of the fluid \\
$\mathbf{I}$ & $3 \times 3$ identity matrix \\
$\mu$ & dynamic viscosity \\
$\alpha_{1}$ & kinematic viscoelasticity parameter \\
$\alpha_{2}$ & cross viscoelasticity parameter \\
$\beta_{1}, \beta_{2}, \beta_{3}$ & third grade material constants \\
$\mathbf{A}_{n}$ & Rivlin-Ericksen kinematic tensor \\
$T$ & transpose operation \\
$p^{*}$ & generalized pressure \\
$B$ & non-dimensional generalized pressure gradient \\
$\lambda$ & Brinkman number \\
$L^{2}(\Omega)$ & space of square integrable functions over the domain $\Omega=(-1,1)$ \\
$H^{1}(\Omega)$ & Sobolev space of $L^{2}-$ functions possessing first order weak derivatives in $L^{2}(\Omega)$ \\
$H_{0}^{1}(\Omega)$ & subspace of compactly supported functions in the usual sense \\
& \\
\hline
\end{tabular}




\subsection{Problem Settings and Governing Equations}

Consider two infinite parallel plates separated apart by a distance $2 L$ where $L>0$, in a Cartesian coordinate system, containing an incompressible steady fluid of grade three between them. Without loss of generality, we assume that the upper plate can be represented by the plane $y=L$, whereas the lower plate can be represented by the plane $y=-L$. Let $\psi$ represent the temperature field and $\mathbf{U}$ the velocity field. Then the governing equations of the flow problem, in the absence of body forces, are

$$
\begin{aligned}
\nabla \cdot \mathbf{U} & =0, & & \text { (Continuity equation) } \\
\rho(\mathbf{U} \cdot \nabla) \mathbf{U} & =\nabla \cdot \sigma, & & \text { (Momentum equation) } \\
k \nabla^{2} \psi+\sigma \cdot \nabla \mathbf{U} & =0, & & \text { (Energy equation) }
\end{aligned}
$$

where $\rho$ is the fluid density, $\sigma$ is the stress tensor, and $k$ is the thermal conductivity of the fluid. The flow is assumed to be unidirectional in the direction of $x$-axis, the upper and the lower plates are maintained at constant temperatures $\psi_{L}$ and $\psi_{-L}$ respectively. Assume that the lower plate is rigid. Moreover the unidirectional velocity field $\mathbf{U}$ and the temperature field $\psi$ depend only on the $y$-coordinate, that is,

$$
\mathbf{U}=u(y) \mathbf{e}_{x} \quad \text { and } \quad \psi=\psi(y)
$$

where $\left(\mathbf{e}_{x}, \mathbf{e}_{y}, \mathbf{e}_{z}\right)$ is the canonical basis of $\mathbb{R}^{3}$; see Figure 1. Following flow configurations are

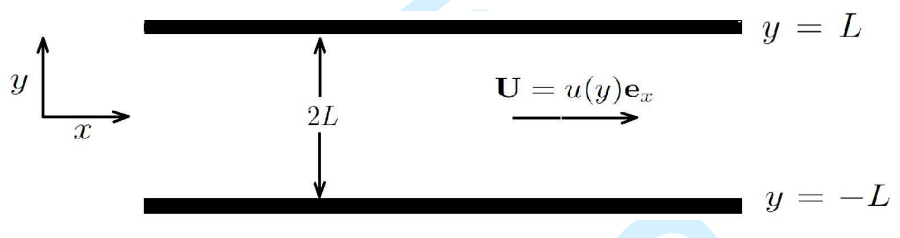

Figure 1: Geometric configuration.

considered.

1. The upper plate is free to move with a constant velocity $\mathbf{U}_{L}$ whereas the pressure gradient on the upper plate is set to be zero (plane Couette flow).

2. The upper plate is also at rest and the fluid is moved by the application of a constant pressure gradient (plane Poiseuille flow).

3. The upper plate moves with constant velocity $\mathbf{U}_{L}=u_{L} \mathbf{e}_{x}$ together with a non-zero constant pressure gradient (generalized Couette flow or the Couette-Poiseuille flow).

\subsection{General Flow Problem}

Recall that the Cauchy stress tensor for a Rivlin-Ericksen fluid of grade three is given by

$$
\sigma=-p \mathbf{I}+\mu \mathbf{A}_{1}+\alpha_{1} \mathbf{A}_{2}+\alpha_{2} \mathbf{A}_{1}^{2}+\beta_{1} \mathbf{A}_{3}+\beta_{2}\left(\mathbf{A}_{1} \mathbf{A}_{2}+\mathbf{A}_{2} \mathbf{A}_{1}\right)+\beta_{3}\left(\operatorname{tr} \mathbf{A}_{2}\right) \mathbf{A}_{1},
$$

see for instance [?], where $t r$ represents the trace of a matrix, $p$ is the hydro-static pressure of the fluid, $\mathbf{I}$ is $3 \times 3$ identity matrix, $\mu$ is the dynamic viscosity, $\alpha_{1}$ and $\alpha_{2}$ are the kinematic 
and cross viscoelasticity parameters and $\beta_{1}, \beta_{2}$ and $\beta_{3}$ are the third grade material constants. Moreover, the Rivlin-Ericksen kinematic tensors $\mathbf{A}_{n}$ for $n=1,2,3, \cdots$, are defined recursively by

$$
\begin{aligned}
& \mathbf{A}_{1}=(\nabla \mathbf{U})^{T}+\nabla \mathbf{U}, \\
& \mathbf{A}_{n}=\frac{\partial \mathbf{A}_{n-1}}{\partial t}+(\mathbf{U} \cdot \nabla) \mathbf{A}_{n-1}+\mathbf{A}_{n-1} \nabla \mathbf{U}+(\nabla \mathbf{U})^{T} \mathbf{A}_{n-1}, \quad n=2,3, \cdots,
\end{aligned}
$$

where $T$ indicates the transpose operation.

By virtue of $(2.1),(2.2),(2.4),(2.5),(2.6)$ and $(2.7)$, we arrive at

$$
\mu \frac{d^{2} u}{d y^{2}}+6\left(\beta_{3}+\beta_{2}\right)\left(\frac{d u}{d y}\right)^{2} \frac{d^{2} u}{d y^{2}}=\frac{\partial p^{*}}{\partial x} \quad \text { and } \quad \frac{\partial p^{*}}{\partial y}=0=\frac{\partial p^{*}}{\partial z},
$$

where $p^{*}$ is the generalized pressure defined by

$$
p^{*}:=p-\left(2 \alpha_{1}+\alpha_{2}\right)\left(\frac{d u}{d y}\right)^{2} .
$$

On the other hand, note that by virtue of (2.4) and (2.5), Equation (2.3) yields

$$
k \frac{d^{2} \psi}{d y^{2}}+\mu\left(\frac{d u}{d y}\right)^{2}+2\left(\beta_{2}+\beta_{3}\right)\left(\frac{d u}{d y}\right)^{4}=0 .
$$

Consequently, the general flow problem takes the form as in [21]

$$
\left\{\begin{array}{l}
\mu \frac{d^{2} u}{d y^{2}}+6\left(\beta_{2}+\beta_{3}\right)\left(\frac{d u}{d y}\right)^{2} \frac{d^{2} u}{d y^{2}}=b, \quad-L<y<L, \\
k \frac{d^{2} \psi}{d y^{2}}+\mu\left(\frac{d u}{d y}\right)^{2}+2\left(\beta_{2}+\beta_{3}\right)\left(\frac{d u}{d y}\right)^{4}=0, \quad-L<y<L \\
\psi(-L)=\psi_{-L}, \quad \psi(L)=\psi_{L}, \\
u(-L)=0, \quad u(L)=a,
\end{array}\right.
$$

where $a, b \in \mathbb{R}$, and the boundary value problem (2.11) corresponds to the plane Couette flow if $a=u_{L}$ and $b=0$, plane Poiseuille flow if $a=0$ and $b \neq 0$, and generalized Couette flow if $a=u_{L}$ and $b \neq 0$.

\subsection{Non-Dimensionalization}

Introduce the following dimensionless variables

$$
\widetilde{y}=\frac{y}{L}, \quad \widetilde{u}=\frac{u}{u_{L}}, \quad \widetilde{\psi}=\frac{\psi-\psi_{-L}}{\psi_{L}-\psi_{-L}},
$$

and characteristic parameters

$$
\beta=\left(\frac{\beta_{2}+\beta_{3}}{\mu}\right)\left(\frac{u_{L}}{L}\right)^{2}, \quad \lambda=\frac{\mu u_{L}^{2}}{k\left(\psi_{-L}-\psi_{L}\right)}, \quad B=-\frac{L^{2}}{\mu u_{L}} \frac{\partial p^{*}}{\partial x} .
$$

The parameter $\beta$ accounts for third grade behavior of the fluid, $B$ is the non-dimensional generalized pressure gradient, whereas $\lambda$ is the so-called Brinkman number. 
By using (2.12) and (2.13) and dropping the superscripts from the dimensionless variables for brevity, the boundary value problem $(2.11)$ can be written in non-dimensional from as

$$
\begin{cases}\frac{d^{2} u}{d y^{2}}+6 \beta\left(\frac{d u}{d y}\right)^{2} \frac{d^{2} u}{d y^{2}}=-B, & -1<y<1 \\ \frac{d^{2} \psi}{d y^{2}}+\lambda\left(\frac{d u}{d y}\right)^{2}+2 \beta \lambda\left(\frac{d u}{d y}\right)^{4}=0, & -1<y<1 \\ \psi(-1)=0, \quad \psi(1)=1 \\ u(-1)=0, \quad u(1)=A\end{cases}
$$

The boundary value problem (2.14) corresponds to plane Couette flow if $A=1$ and $B=0$, plane Poiseuille flow if $A=0$ and $B \neq 0$, and generalized Couette flow if $A=1$ and $B \neq 0$. Dimensionless skin friction coefficients and local Nusselt numbers are given by

$$
\begin{gathered}
\frac{\operatorname{Re} C_{f_{1}}}{2}=\left[\frac{d^{2} u}{d y^{2}}+2 \beta \operatorname{Re}\left(\frac{d^{2} u}{d y^{2}}\right)^{3}\right]_{y=1}, \\
\frac{\operatorname{Re} C_{f_{2}}}{2}=\left[\frac{d^{2} u}{d y^{2}}+2 \beta R e\left(\frac{d^{2} u}{d y^{2}}\right)^{3}\right]_{y=-1}, \\
\frac{N u_{1}}{R e}=-\left(\frac{d \psi}{d y}\right)_{y=1} \text { and } \frac{N u_{2}}{R e^{2}}=-\left(\frac{d \psi}{d y}\right)_{y=-1},
\end{gathered}
$$

where $R e=\frac{A L}{\nu}$.

\section{Numerical Solution Using FEM}

In this section, we shall provide the numerical solutions to the non-dimensional model problem. Due to inadequacy of the asymptotic techniques for strong non-linearity and large values of parameters, Mahmood et al. [20] attempted to resolve (2.11) by using a Lagrange-Galerkin finite element method.

This method normally solves linear and nonlinear partial differential equations (PDEs) of even order, particularly second order initial and boundary values problems. It transforms the given boundary value problem into a weak formulation by multiplying with a test function on both sides. The weak form includes natural boundary conditions of the problem. The domain of the underlying problem is discretized into smaller disjoint sub-domains, called finite elements or simply elements, which are connected with each other at the boundary of each element. In one dimension the sub-domain elements, also called domain mesh or simply mesh, are intervals. Two dimensional elements are triangles or quadrilaterals or tetrahedrons, or hexagons. The solution to the underlying problem is considered as linear combination of test or shape functions and unknown coefficients within each element. The shape functions may be chosen as polynomials of degree less than or equal to some fixed positive integer having certain properties. The given PDE is then solved over each element and connected at boundary of each element to get the solution of the given PDE for complete domain. The elements may be combined in various ways, so that elements can be used to discretize very complex shape domains, for more details, see [27]. Mahmood et al. [20] used linear polynomials as interpolation functions to obtain a discrete solution. Unfortunately, the general heat flow model (2.14) presented in $\S 2.3$ contains 
nonlinear term wherein second derivative is involved. Consequently, linear polynomials are inadequate to be used as interpolation functions to approximate the solution since their first derivatives are constants.

Remark that the generalized Couette flow model is the most general, whereas plane Couette and plane Poiseuille flows can be regarded as special cases, so we shall present the numerical procedures for the generalized Couette flow in the sequel.

\subsection{Weak Formulation}

We shall denote the space of square integrable functions over the domain $\Omega=(-1,1)$ by $L^{2}(\Omega)$, equipped with the $L^{2}$-inner product and induced $L^{2}$-norm defined respectively by

$$
(u, v)_{L^{2}(\Omega)}=\int_{\Omega} u(x) v(x) d x, \quad \text { and } \quad\|u\|_{L^{2}(\Omega)}=\left(\int_{\Omega}|u(x)|^{2} d x\right)^{1 / 2}, \quad \forall u, v \in L^{2}(\Omega) .
$$

Let us define the Sobolev space $H^{1}(\Omega)$ of $L^{2}$-functions possessing first order weak derivatives in $L^{2}(\Omega)$ and its subspace $H_{0}^{1}(\Omega)$ of compactly supported functions in the usual sense. Recall that these spaces are equipped with following norms and inner products; see for instance $[26]$

$$
\begin{gathered}
(u, v)_{H^{1}(\Omega)}=\int_{\Omega}(u v+\nabla u \cdot \nabla v) d x, \quad \text { and }\|u\|_{H^{1}(\Omega)}=\left(\int_{\Omega}\left(|u|^{2}+|\nabla u|^{2}\right) d x\right)^{1 / 2}, \\
(u, v)_{H_{0}^{1}(\Omega)}=\int_{\Omega} \nabla u \cdot \nabla v d x, \quad \text { and } \quad\|u\|_{H_{0}^{1}(\Omega)}=\left(\int_{\Omega}|\nabla u|^{2} d x\right)^{1 / 2} .
\end{gathered}
$$

Now, consider the generalized Couette flow model

$$
\left\{\begin{array}{l}
\frac{d^{2} u}{d y^{2}}+6 \beta\left(\frac{d u}{d y}\right)^{2} \frac{d^{2} u}{d y^{2}}+B=0, \quad-1<y<1 \\
\frac{d^{2} \psi}{d y^{2}}+\lambda\left(\frac{d u}{d y}\right)^{2}+2 \beta \lambda\left(\frac{d u}{d y}\right)^{4}=0, \quad-1<y<1 \\
\psi(-1)=0, \quad \psi(1)=1 \\
u(-1)=0, \quad u(1)=1 .
\end{array}\right.
$$

Define

$$
\omega(y)=u(y)-u_{E}(y) \quad \text { and } \quad \gamma(y)=\psi(y)-\psi_{E}(y),
$$

where $u_{E}(y)=\frac{y+1}{2}=\psi_{E}(y)$. Using the transformations (3.5), the boundary value problem (3.4) takes on the form

$$
\left\{\begin{array}{l}
\frac{d^{2} \omega}{d y^{2}}+\mathcal{N}_{1}\left(\omega, \omega^{\prime}, \omega^{\prime \prime}\right)=C_{1} \\
\frac{d^{2} \gamma}{d y^{2}}+\mathcal{N}_{2}\left(\omega, \omega^{\prime}\right)=C_{2} \\
\omega(-1)=0=\omega(1) \\
\gamma(-1)=0=\gamma(1)
\end{array}\right.
$$


where $\alpha=1+3 / 2 \beta, C_{1}=\frac{B}{\alpha}$, and $C_{2}=\frac{\lambda}{4}$. Here $\mathcal{N}_{1}\left(\omega, \omega^{\prime}, \omega^{\prime \prime}\right)$ and $\mathcal{N}_{2}\left(\omega, \omega^{\prime}\right)$ are nonlinear operators defined by

$$
\begin{aligned}
\mathcal{N}_{1}\left(\omega, \omega^{\prime}, \omega^{\prime \prime}\right) & :=\frac{6 \beta}{\alpha}\left(\frac{d \omega}{d y}\right)^{2}\left(\frac{d^{2} \omega}{d y^{2}}\right)+\frac{6 \beta}{\alpha}\left(\frac{d \omega}{d y}\right)\left(\frac{d^{2} \omega}{d y^{2}}\right), \\
\mathcal{N}_{2}\left(\omega, \omega^{\prime}\right) & :=\lambda\left(\frac{d \omega}{d y}\right)^{2}+\lambda\left(\frac{d \omega}{d y}\right)+2 \beta \lambda\left(\frac{d \omega}{d y}+\frac{1}{2}\right)^{4}
\end{aligned}
$$

with $\beta \neq-2 / 3$.

Therefore, we have the following weak formulation of the boundary vale problem (3.6):

Weak Problem 3.1. Find $(w, \gamma) \in H_{0}^{1}(\Omega) \times H_{0}^{1}(\Omega)$ such that for all $(v, z) \in H_{0}^{1}(\Omega) \times H_{0}^{1}(\Omega)$,

$$
\begin{gathered}
-\int_{-1}^{1}\left(\frac{d \omega}{d y}\right)\left(\frac{d v}{d y}\right) d y+\int_{-1}^{1} \mathcal{N}_{1}\left(\omega, \omega^{\prime}, \omega^{\prime \prime}\right) v(y) d y=C_{1} \int_{-1}^{1} v(y) d y \\
-\int_{-1}^{1}\left(\frac{d \gamma}{d y}\right)\left(\frac{d z}{d y}\right) d y+\int_{-1}^{1} \mathcal{N}_{2}\left(\omega, \omega^{\prime}\right) z(y) d y=C_{2} \int_{-1}^{1} z(y) d y
\end{gathered}
$$

The next subsection is dedicated to employ a finite element method in order to obtain an approximate solution to the flow problem (3.6).

\subsection{Finite Element Approximations}

Let $-1=y_{1}<y_{2}<\cdots<y_{n}<y_{n+1}=1$. Define the partition of the domain $\Omega$ into $n$ subdomains $\Omega_{i}$ for $i=1,2, \ldots, n$ such that

$$
\bar{\Omega}=\cup_{i=1}^{n} \bar{\Omega}_{i}, \quad \Omega_{i} \cap \Omega_{j}=\emptyset, \quad \forall i \neq j,
$$

where

$$
\Omega_{i}=\left(y_{i}, y_{i+1}\right), \quad i, j=1,2, \ldots, n .
$$

Let $h_{i}$ be the length of element $\Omega_{i}$, that is

$$
h_{i}:=y_{i+1}-y_{i}=h:=\frac{2}{n+1}, \quad i=1,2, \ldots, n .
$$

Define a sequence of finite dimensional approximation subspace $V_{0}^{h}(\Omega)$ of $H_{0}^{1}(\Omega)$ by

$$
V_{0}^{h}=\left\{w \in H_{0}^{1}(\Omega)|w|_{\Omega_{i}} \in P_{k}\left(\Omega_{i}\right), \forall i=1,2, \cdots, n\right\}
$$

where $P_{k}\left(\Omega_{i}\right)$ is a finite element interpolating space of polynomials with degree $\leq k$ over the element $\Omega_{i}$ for each $i=1,2, \cdots, n$. If we interpolate the unknown functions $w$ and $\gamma$ over the element $\Omega_{i}$ with nodes $n_{e}$ using polynomials of degree $k$, then the approximate solution over each element $\left(w_{h}^{i}, \gamma_{h}^{i}\right) \in V_{0}^{h} \times V_{0}^{h}$ can be written as

$$
w_{h}^{i}(y)=\sum_{j=1}^{n_{e}} w_{j}^{i} N_{j}^{i}(y), \quad \text { and } \quad \gamma_{h}^{i}(y)=\sum_{j=1}^{n_{e}} \gamma_{j}^{i} N_{j}^{i}(y), \quad y \in \bar{\Omega}_{i}=\left[y_{i}, y_{i+1}\right],
$$

where $w_{j}^{i}$ and $\gamma_{j}^{i}$ are unknown function values at node $y_{j}$ for $j=1,2, \cdots, n_{e}$ and $N_{j}^{i}$ are polynomials of degree $k$ associated with node $y_{j}$ over the element $\Omega_{i}$.

With the help of Equation (3.13), we can write the finite element approximation of the problem (3.9) as below: 
Dicrete Weak Problem 3.2. Find $\left(w_{h}^{i}, \gamma_{h}^{i}\right) \in V_{0}^{h}(\Omega) \times V_{0}^{h}(\Omega)$ such that

$$
\begin{gathered}
-\int_{y_{i}}^{y_{i+1}} \frac{d \omega_{h}^{i}}{d y} \cdot \frac{d v_{h}}{d y} d y+\int_{y_{i}}^{y_{i+1}} \mathcal{N}_{1}\left(\omega_{h}^{i},\left(\omega_{h}^{i}\right)^{\prime},\left(\omega_{h}^{i}\right)^{\prime \prime}\right) v_{h}(y) d y=C_{1} \int_{y_{i}}^{y_{i+1}} v_{h}(y) d y, \\
-\int_{y_{i}}^{y_{i+1}} \frac{d \gamma_{h}^{i}}{d y} \cdot \frac{d z_{h}}{d y} d y+\int_{y_{i}}^{y_{i+1}} \mathcal{N}_{2}\left(\omega_{h}^{i},\left(\omega_{h}^{i}\right)^{\prime}\right) z_{h}(y) d y=C_{2} \int_{y_{i}}^{y_{i+1}} z_{h}(y) d y
\end{gathered}
$$

for all $\left(v_{h}, z_{h}\right) \in V_{0}^{h}(\Omega) \times V_{0}^{h}(\Omega)$.

Remark that when the polynomials of degree two are used as interpolation functions to approximate the solution on each element, Equations (3.13) take on the form

$$
\begin{gathered}
w_{h}^{i}(y)=w_{1}^{i} N_{1}^{i}(y)+w_{2}^{i} N_{2}^{i}(y)+w_{3}^{i} N_{3}^{i}(y), \quad y \in \bar{\Omega}_{i}=\left[y_{i}, y_{i+1}\right] \\
\gamma_{h}^{i}(y)=\gamma_{1}^{i} N_{1}^{i}(y)+\gamma_{2}^{i} N_{2}^{i}(y)+\gamma_{3}^{i} N_{3}^{i}(y), \quad y \in \bar{\Omega}_{i}=\left[y_{i}, y_{i+1}\right] .
\end{gathered}
$$

Equivalently, the above equations can be written in a matrix form as

$$
\begin{aligned}
& w_{h}^{i}(y)=\left[\begin{array}{lll}
N_{1}^{i}(y) & N_{2}^{i}(y) & N_{3}^{i}(y)
\end{array}\right]\left[\begin{array}{c}
w_{1}^{i} \\
w_{2}^{i} \\
w_{3}^{i}
\end{array}\right]=\mathbf{N}_{i}^{T} \mathbf{W}_{i}, \quad y \in \bar{\Omega}_{i}=\left[y_{i}, y_{i+1}\right] \\
& \gamma_{h}^{i}(y)=\left[\begin{array}{lll}
N_{1}^{i}(y) & N_{2}^{i}(y) & N_{3}^{i}(y)
\end{array}\right]\left[\begin{array}{c}
\gamma_{1}^{i} \\
\gamma_{2}^{i} \\
\gamma_{3}^{i}
\end{array}\right]=\mathbf{N}_{i}^{T} \mathbf{\Gamma}_{i}, \quad y \in \bar{\Omega}_{i}=\left[y_{i}, y_{i+1}\right],
\end{aligned}
$$

where

$$
\mathbf{W}_{i}=\left[\begin{array}{c}
w_{1}^{i} \\
w_{2}^{i} \\
w_{3}^{i}
\end{array}\right], \quad \boldsymbol{\Gamma}_{i}=\left[\begin{array}{c}
\gamma_{1}^{i} \\
\gamma_{2}^{i} \\
\gamma_{3}^{i}
\end{array}\right], \quad \mathbf{N}_{i}=\left[\begin{array}{c}
N_{1}^{i}(y) \\
N_{2}^{i}(y) \\
N_{3}^{i}(y)
\end{array}\right] .
$$

Making use of Equations (3.16) in Equations (3.14), we obtain

$$
\begin{gathered}
-\mathbf{A}_{1}^{i} \mathbf{W}_{i}+\mathcal{N}_{1}^{i}\left(\mathbf{W}_{i}, \mathbf{N}_{i}\right)=\mathbf{B}_{1}^{i}\left(\mathbf{W}_{i}\right), \\
-\mathbf{A}_{2}^{i} \boldsymbol{\Gamma}_{i}+\mathcal{N}_{2}^{i}\left(\mathbf{W}_{i}, \mathbf{N}_{i}\right)=\mathbf{B}_{2}^{i}\left(\mathbf{W}_{i}\right),
\end{gathered}
$$

where, for $j=1,2$,

$$
\mathbf{A}_{j}^{i}=\left(\begin{array}{ccc}
\int_{y_{i}}^{y_{i+1}}\left(N_{1}^{i}\right)^{\prime}\left(N_{1}^{i}\right)^{\prime} d y & \int_{y_{i}}^{y_{i+1}}\left(N_{1}^{i}\right)^{\prime}\left(N_{2}^{i}\right)^{\prime} d y & \int_{y_{i}}^{y_{i+1}}\left(N_{1}^{i}\right)^{\prime}\left(N_{3}^{i}\right)^{\prime} d y \\
\int_{y_{i}}^{y_{i+1}}\left(N_{2}^{i}\right)^{\prime}\left(N_{1}^{i}\right)^{\prime} d y & \int_{y_{i}}^{y_{i+1}}\left(N_{2}^{i}\right)^{\prime}\left(N_{2}^{i}\right)^{\prime} d y & \int_{y_{i}}^{y_{i+1}}\left(N_{2}^{i}\right)^{\prime}\left(N_{3}^{i}\right)^{\prime} d y \\
\int_{y_{i}}^{y_{i+1}}\left(N_{3}^{i}\right)^{\prime}\left(N_{1}^{i}\right)^{\prime} d y & \int_{y_{i}}^{y_{i+1}}\left(N_{3}^{i}\right)^{\prime}\left(N_{2}^{i}\right)^{\prime} d y & \int_{y_{i}}^{y_{i+1}}\left(N_{3}^{i}\right)^{\prime}\left(N_{3}^{i}\right)^{\prime} d y
\end{array}\right)
$$

and

$$
\mathcal{N}_{j}^{i}\left(\mathbf{W}_{i}, \mathbf{N}_{i}\right)=\left(\begin{array}{c}
\int_{y_{i}}^{y_{i+1}} \mathcal{N}_{j}^{i} N_{1}^{i} d y \\
\int_{y_{i}}^{y_{i+1}} \mathcal{N}_{j}^{i} N_{2}^{i} d y \\
\int_{y_{i}}^{y_{i+1}} \mathcal{N}_{j}^{i} N_{3}^{i} d y
\end{array}\right), \quad \mathbf{B}_{j}^{i}\left(\mathbf{N}_{i}\right)=\left(\begin{array}{c}
C_{j} \int_{y_{i}}^{y_{i+1}} N_{1}^{i} d y \\
C_{j} \int_{y_{i}}^{y_{i+1}} N_{2}^{i} d y \\
C_{j} \int_{y_{i}}^{y_{i+1}} N_{3}^{i} d y
\end{array}\right) .
$$

Refer to the Appendix A for explicit expressions of $P_{2}$-basis functions $N_{j}^{i}$ and corresponding element matrices $\mathbf{A}_{j}^{i}$, non-linear load matrices $\mathcal{N}_{j}^{i}$ and right hand side matrices $\mathbf{B}_{j}^{i}$. 


\subsection{Implementation Details}

In this section, we present a numerical scheme for the resolution of the matrix equations given by (3.17.) As, the matrix equations (3.17) form nonlinear systems in $w_{i}^{\prime} s$ and $\gamma_{i}^{\prime} s$, Newton's iterative method will be evoked in order to obtain approximate solutions. The following algorithm explains the basic implementation scheme.

\subsubsection{Numerical Algorithm}

i. Initialize the input parameters involved in the matrix equations, e.g., $\beta, \lambda$.

ii. Create a mesh with $n$ elements $\Omega_{i}, i=1,2, \ldots, n$ having $n+1$ nodes.

iii. Allocate memory for the global matrices $\mathbf{A}_{1}, \mathbf{A}_{2}$ of order $(n+1) \times(n+1)$, nonlinear vectors $\mathcal{N}_{1}, \mathcal{N}_{2}$ and the right hand side vectors $\mathbf{B}_{1}, \mathbf{B}_{2}$ of order $(n+1) \times 1$ and initialize all matrix entries to zero.

iv. Initialize and start While loop for Newton's iterations.

a. For $i=1,2,3, \ldots, n$, do compute the element stiffness matrices $\mathbf{A}_{1}^{i}, \mathbf{A}_{2}^{i}$, nonlinear vectors $\mathcal{N}_{1}^{i}, \mathcal{N}_{2}^{i}$ and right hand side vectors $\mathbf{B}_{1}^{i}, \mathbf{B}_{2}^{i}$.

b. Add element matrices into the corresponding global matrices at respective positions.

c. Apply the Newton's iteration to update the solutions and terminate the while loop.

v. Apply boundary conditions.

vi. Plot the solutions.

Following the steps mentioned in the algorithm, we build a code in MatLab in order to carry out the simulations.

\section{Numerical Simulations and Discussion}

In this section, numerical error estimates are presented and numerical results for heat flow profile are delineated in order to highlight the influence of pertinent flow parameters on heat transfer for different flow configurations.

\subsection{Numerical Error Estimates}

In order to validate the numerical scheme build in previous section, it is important to perform the numerical error analysis and check the order of convergence of the numerical scheme. Theoretically, the numerical error estimates for the problems of the kind (3.6), using Lagrange interpolation functions of degree $k$, are given by

$$
\begin{aligned}
&\left\|w-w_{e x}\right\|_{L^{2}(\Omega)} \leqq c_{1} h^{k+1}, \\
&\left\|\psi-\psi_{e x}\right\|_{L^{2}(\Omega)} \leqq c_{2} h^{k+1},
\end{aligned}
$$

refer to [27]. Here $(w, \psi)$ and $\left(w_{e x}, \psi_{e x}\right)$ are the approximate and exact solutions of the flow problem respectively with $c_{1}$ and $c_{2}$ being the constants independent of $h$. 


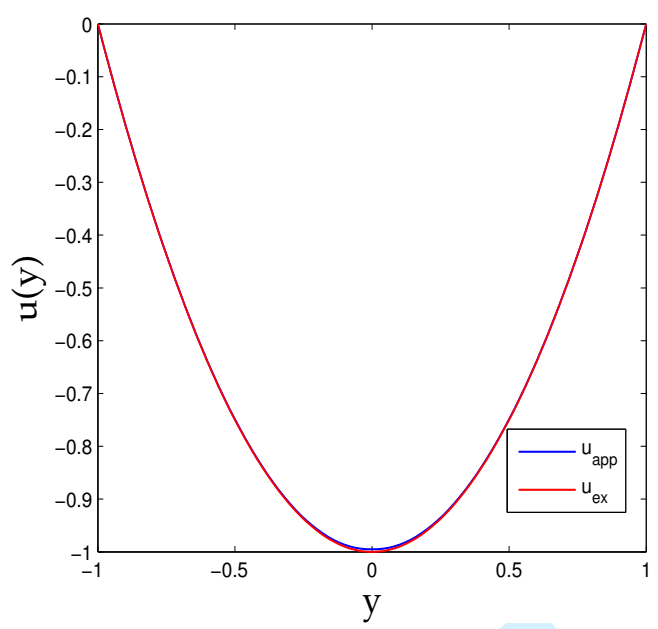

(a) Exact and approximate velocity profile

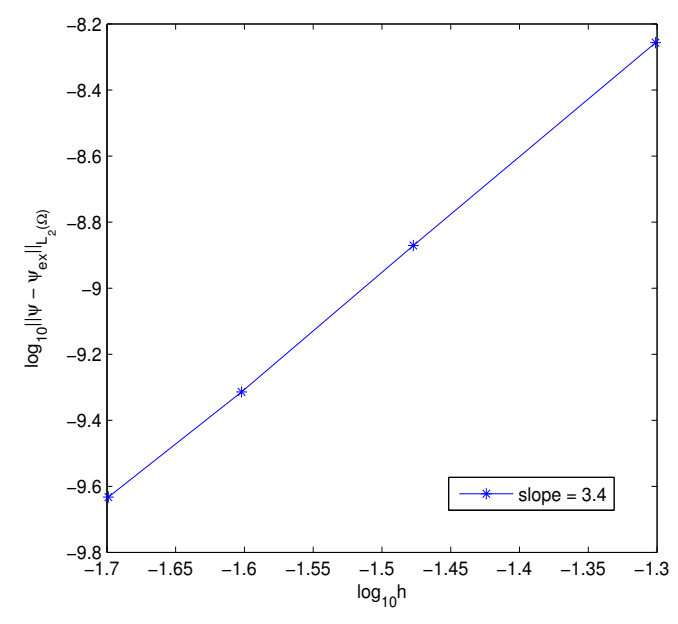

(b) Error curve for temperature profile

Figure 2: Error estimates for velocity and temperature fields.

In order to study the convergence of numerical scheme, we have fabricated the exact solution by adding an arbitrary function on the right-hand side of the problem under consideration. In order to validate our numerical scheme, we have performed two types of computations. First, we have computed the numerical solution of the problem and compared it to the fabricated exact solution. We have shown the comparison of numerically approximated and exact solution of the velocity in Figure 2(a). Second, by varying the step size, $h$, in the numerical scheme, we have evaluated the norms $\left\|w-w_{e x}\right\|_{L^{2}(\Omega)}$ and $\left\|\psi-\psi_{e x}\right\|_{L^{2}(\Omega)}$. In the Figure 2(b), we plot the error curve for temperature in $\log$ scales of $L^{2}$-norm versus step size $h$. It can be seen that the slope of the error curve is approximately equal to 3 in agreement to the theoretical error estimates; see (4.1b). It indicates that our numerical scheme is convergent with order of convergence $O\left(h^{3}\right)$ in $L^{2}$-norm. Both kind of tests demonstrate that our numerical scheme is consistent and convergent. Therefore, we can further perform the realistic physical simulations of the flow problem (3.6).

\subsection{Influence of Flow Parameters}

This section is in order to study behavior of flow with respect to third grade flow parameter, Brinkman number and pressure gradient.

\subsubsection{Couette flow}

Remark that for Couette flow, exact velocity and temperature fields can be obtained explicitly, refer to [24], as

$$
u(y)=\frac{1}{2}(1+y) \quad \text { and } \quad \psi(y)=-\frac{-\lambda}{16} y^{2}(2+\beta)+\frac{y}{2}+\frac{1}{16}((8+\lambda(2+\beta)) .
$$

In Figure 3, the influence of third grade flow parameter $\beta$ on the velocity and temperature field is depicted, fixing the Brinkman number $\lambda=1$. As expected from the knowledge of exact 
solution (4.2), the velocity is a linear function of depth $y$ and is independent on $\beta$. Figure 3(b) indicates that the increase in the temperature is directly proportional to increase in $\beta$. As there are no à priori assumptions on the smallness of third grade parameter $\beta$, the results are also valid for its large values.

Figure is sketched to analyze the behavior of third grade parameter $\beta$ on the velocity profile. It is perceived that velocity profile increases while corresponding boundary layer thickness decreases with the increase of $\beta$. This is due to the fact that third grade parameter is inversely proportional to the fluid viscosity. As $\beta$ increases viscosity of the fluid decreases which enhances velocity profile. In Figure 4, we provide the dependence of temperature field on the Brinkman number $\lambda$ for a fixed third grade parameter $\beta=1$. The numerical results indicate that the Brinkman number directly increases the temperature.

Opposite effects are observed for porosity parameter $\lambda$. It is cleared from the figure that with the increases of $\lambda$ velocity profile decreases while momentum boundary layer thickness increases. As $\lambda$ increases, porosity of the medium and viscosity of the fluid increase. Hence velocity profile decreases.

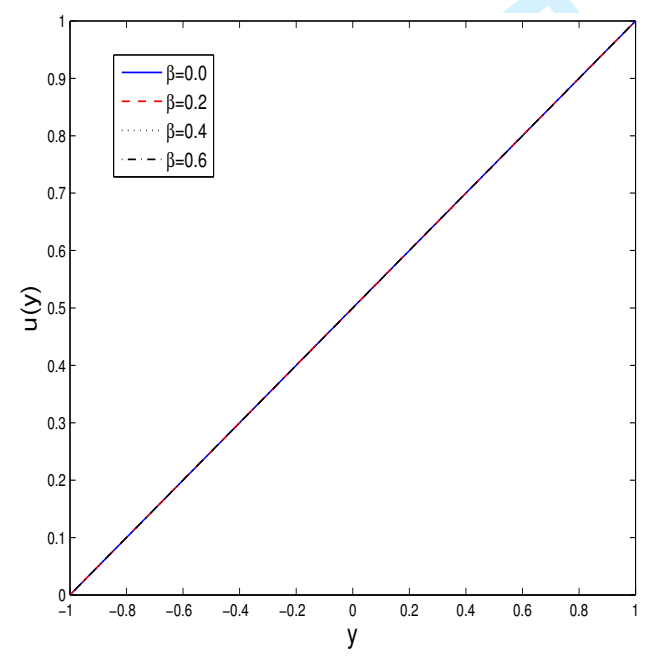

(a) Velocity field

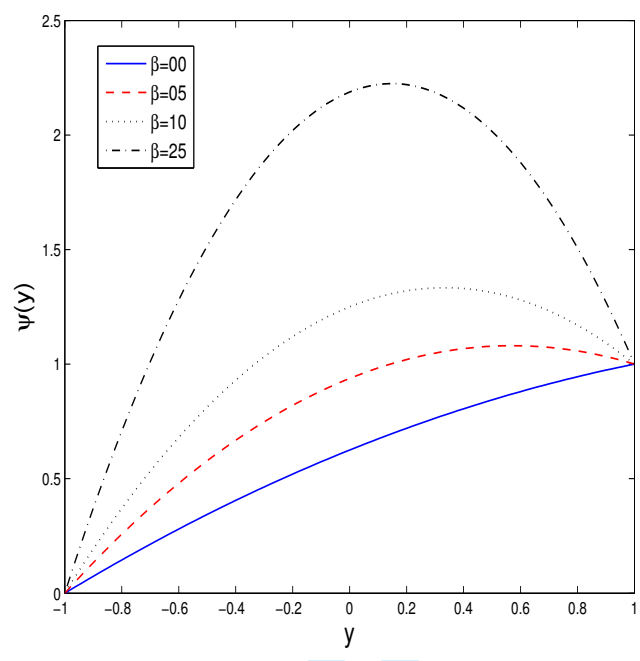

(b) Temperature field

Figure 3: Influence of $\beta$ on the velocity and temperature for plane Couette flow.

\subsubsection{Poiseuille flow}

In Figure 5, we illustrate velocity and temperature fields for four different values of third grade material parameter $\beta$ in plane Poiseuille flow setting. In order to depict velocity variations with respect to $\beta$ we take $\lambda=1$ and $B=1$ whereas for temperature variations we take $B=5$. It is substantiated that the increase in the velocity is directly proportional to the increase in $\beta$. A similar behavior is observed for temperature with respect to $\beta$. The results agree with those provided in [20].

In plane Poiseuille flow, it can be observed in Figure 6 that the velocity profile is strongly influenced by the non-dimensional generalized pressure gradient $B$. However, on contrary, temperature is weakly dependent on $B$. Both temperature and velocity decrease with increasing 


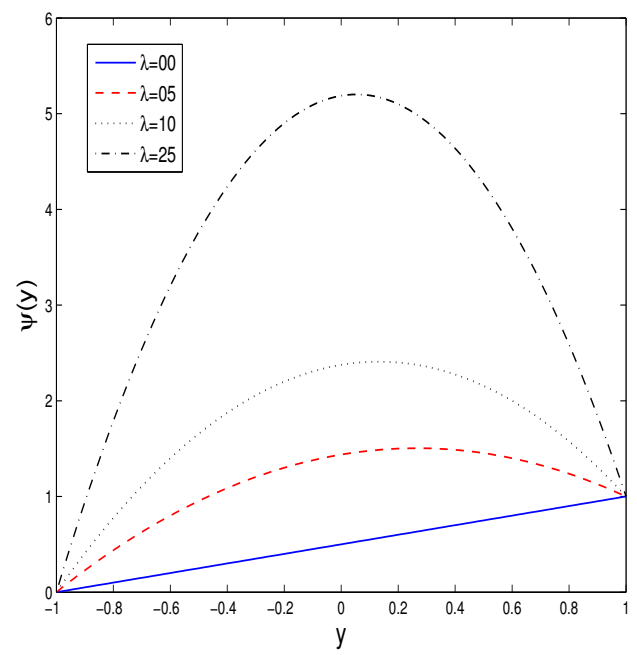

Figure 4: Influence of $\lambda$ on the temperature field in plane Couette Flow.

value of $B$. The other parameters are taken to be $\beta=1$ and $\lambda=1$.

Finally, the dependency of temperature on Brinkman number $\lambda$ is assessed in Figure 7 , and it is found that the increase in temperature is inversely proportional to that of $\lambda$. To get these results, we have fixed the parameters $B=1$ and $\beta=1$.

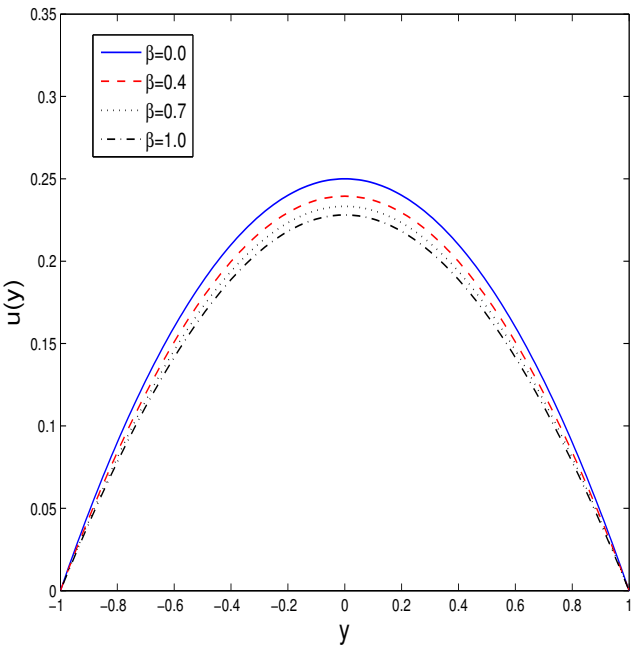

(a) Velocity field

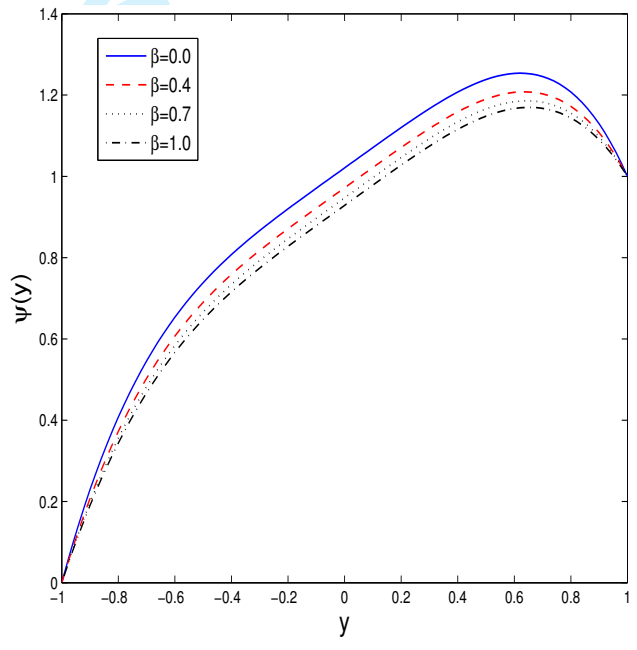

(b) Temperature field

Figure 5: Influence of $\beta$ on the velocity and temperature in plane Poiseuille Flow. 


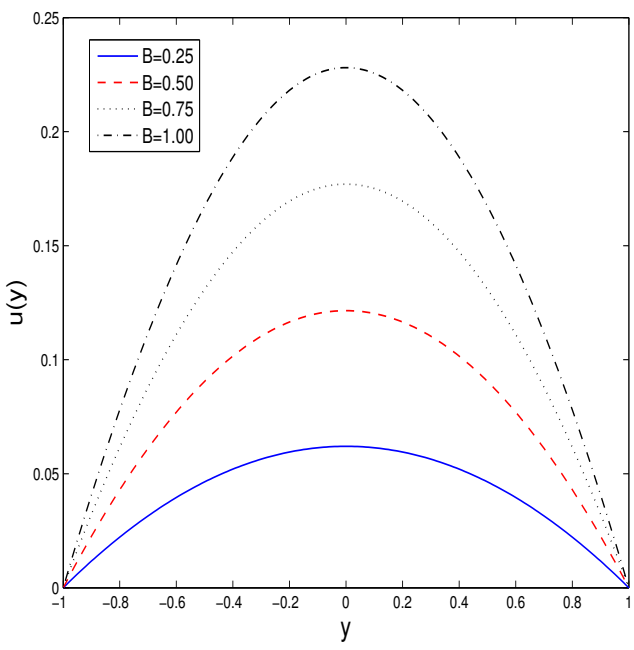

(a) Velocity field

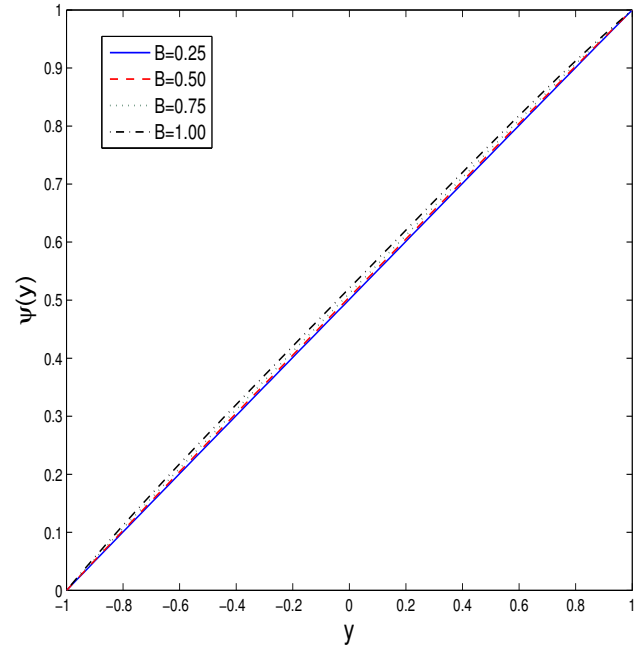

(b) Temperature field

Figure 6: Influence of $B$ on the velocity and temperature in Poiseuille Flow.

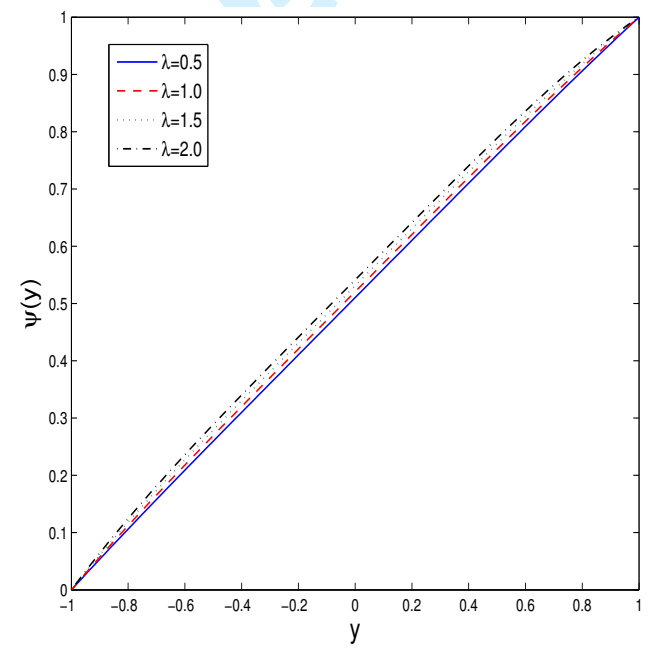

Figure 7: Influence of $\lambda$ on the temperature in plane Poiseuille Flow.

Table 1: Comparison of results obtained for plane Poiseuille flow using various methods when $B=-1$ and $\beta=0.1$.

\begin{tabular}{|c|c|c|c|c|c|c|}
\hline $\mathbf{y}$ & NUM & HPM & HAM & DTM & MDTM & FEM \\
\hline 0.00 & 0.462906 & 0.466048 & 0.463219 & 0.462906 & 0.462906 & 0.462916 \\
0.10 & 0.457911 & 0.461053 & 0.458224 & 0.457736 & 0.457887 & 0.457912 \\
\hline \multicolumn{3}{|c|}{} & \multicolumn{5}{|c|}{ Continued on next page } \\
\hline
\end{tabular}


Table 1 - continued from previous page

\begin{tabular}{|c|l|l|l|l|l|l|}
\hline $\mathbf{y}$ & NUM & HPM & HAM & DTM & MDTM & \multicolumn{1}{|c|}{ FEM } \\
\hline 0.20 & 0.442985 & 0.446127 & 0.443298 & 0.441523 & 0.442887 & 0.442995 \\
0.30 & 0.418297 & 0.421439 & 0.418610 & 0.413160 & 0.418079 & 0.418307 \\
0.40 & 0.384111 & 0.387253 & 0.384424 & 0.371472 & 0.383729 & 0.384121 \\
0.50 & 0.340758 & 0.343900 & 0.341072 & 0.315181 & 0.340174 & 0.340768 \\
0.60 & 0.288613 & 0.291754 & 0.288926 & 0.242802 & 0.287792 & 0.288623 \\
0.70 & 0.228072 & 0.231200 & 0.228379 & 0.152413 & 0.226983 & 0.228082 \\
0.80 & 0.159532 & 0.162567 & 0.159812 & 0.041245 & 0.158150 & 0.159542 \\
0.90 & 0.083384 & 0.085884 & 0.083577 & -0.095011 & 0.081686 & 0.083394 \\
1.00 & 0 & 0 & 0 & -0.263253 & -0.0020344 & 0 \\
\hline
\end{tabular}

\subsubsection{Generalized Couette flow}

The dependence of velocity and temperature on the parameter $\beta$, the generalized pressure gradient in non-dimensional form, $B$, and Brinkman number $\lambda$ is delineated in Figures 8, 9 and 10 respectively in generalized Couette flow. The velocity profile shows a similar behavior as in plane Poiseuille flow with respect to $\beta$, however, temperature profile shows an opposite behavior from that in plane Poiseuille flow, see Figures 8(a) and 8(a). The pressure gradient $B$ and the Brinkman number $\lambda$ show a similar influence on velocity and temperature profile as observed in plane Poiseuille flow, refer to Figures 9(a), 9(b) and 10.

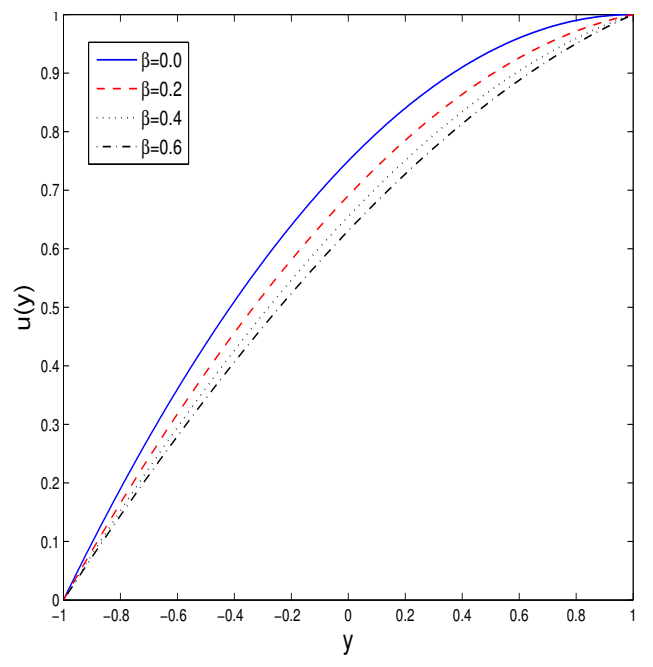

(a) Velocity field

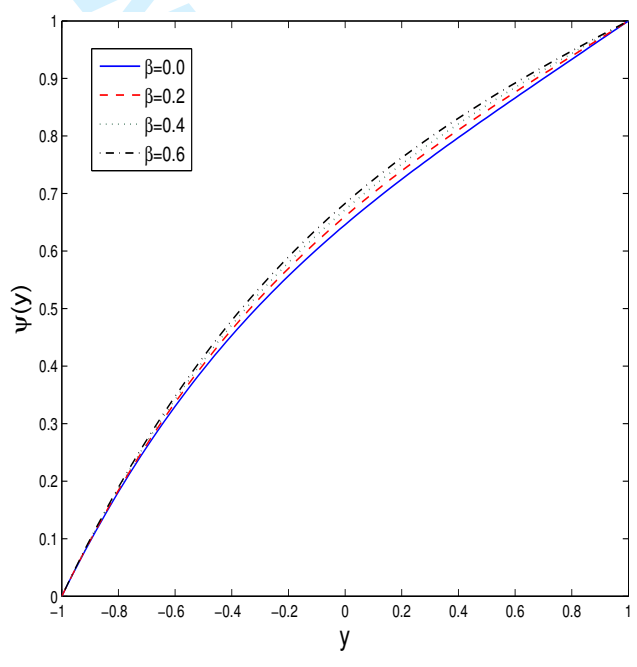

(b) Temperature field

Figure 8: Influence of $\beta$ on the velocity and temperature fields in generalized Couette Flow 


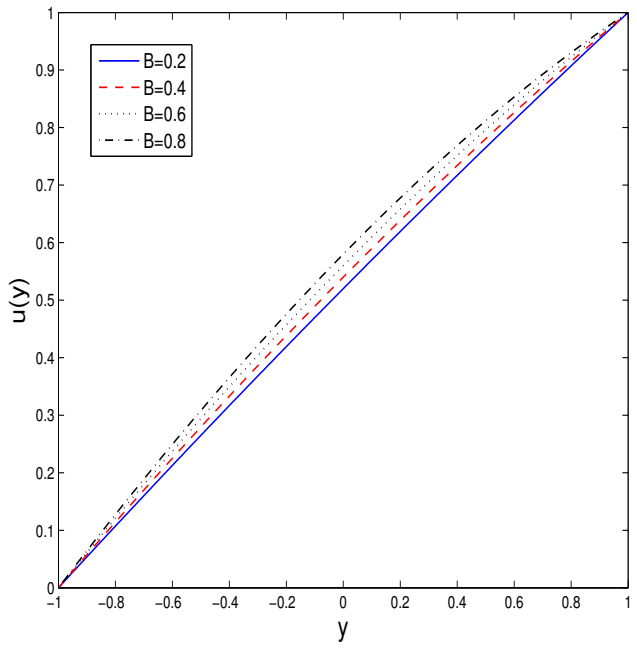

(a) Velocity field

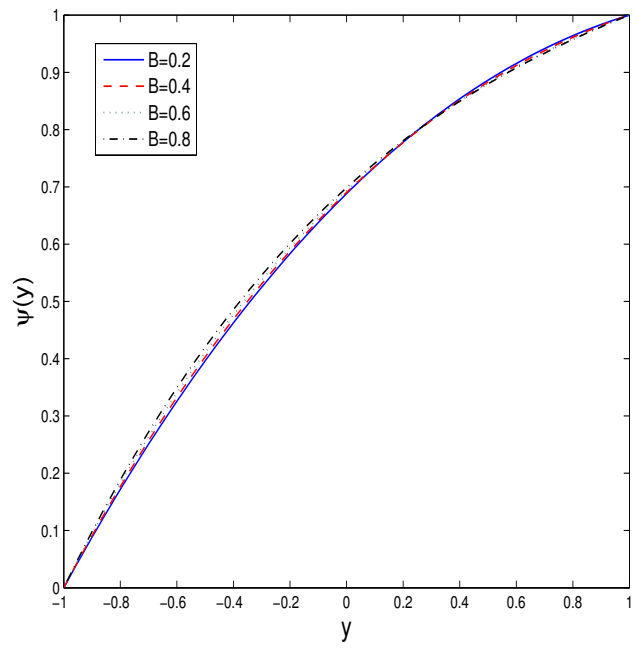

(b) Temperature field

Figure 9: Influence of $B$ on the velocity and temperature in generalized Couette Flow

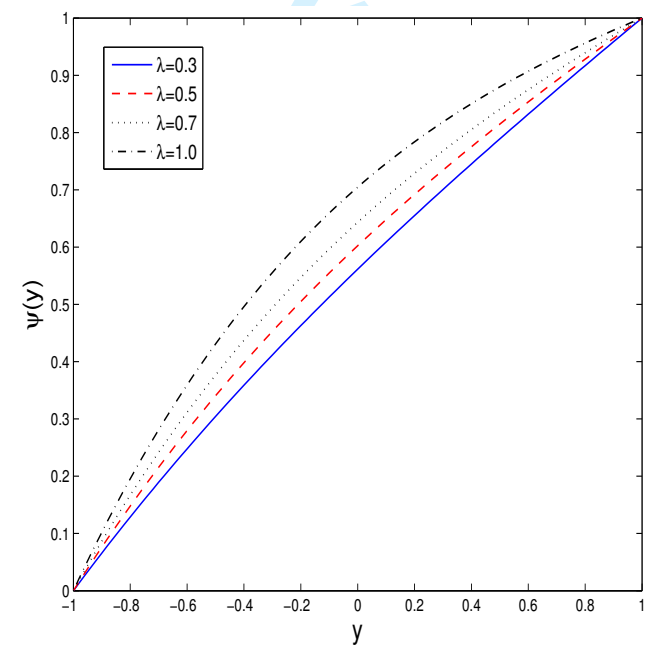

Figure 10: Influence of $\lambda$ on the temperature in generalized Couette Flow

Table 2: Comparison of results obtained for generalized Couette flow using various methods when $B=-1$ and $\beta=0.3$.

\begin{tabular}{|c|c|c|c|c|c|c|}
\hline $\mathbf{y}$ & NUM & HPM & HAM & DTM & MDTM & FEM \\
\hline 0.00 & 0 & 0 & 0 & 0 & 0 & 0 \\
\hline
\end{tabular}


Table 2 - continued from previous page

\begin{tabular}{|c|l|l|l|l|l|l|}
\hline $\mathbf{y}$ & \multicolumn{1}{|c}{ NUM } & \multicolumn{1}{|c|}{ HPM } & \multicolumn{1}{|c|}{ HAM } & \multicolumn{1}{|c|}{ DTM } & \multicolumn{1}{|c|}{ MDTM } & \multicolumn{1}{|c|}{ FEM } \\
\hline 0.10 & 0.1151930 & 0.09460610 & 0.1151830 & 0.1151410 & 0.1151860 & 0.108996 \\
0.20 & 0.227380 & 0.184952 & 0.227359 & 0.226923 & 0.22735 & 0.216126 \\
0.30 & 0.336444 & 0.270894 & 0.336413 & 0.334751 & 0.336374 & 0.321342 \\
0.40 & 0.442259 & 0.351905 & 0.442217 & 0.437799 & 0.442131 & 0.424597 \\
0.50 & 0.544686 & 0.427254 & 0.544631 & 0.534872 & 0.54448 & 0.525840 \\
0.60 & 0.643572 & 0.495862 & 0.643503 & 0.624142 & 0.643265 & 0.625020 \\
0.70 & 0.738746 & 0.554875 & 0.738661 & 0.702659 & 0.738315 & 0.722082 \\
0.80 & 0.83002 & 0.595863 & 0.829913 & 0.765482 & 0.829438 & 0.816972 \\
0.90 & 0.917184 & 0.63878 & 0.917051 & 0.804169 & 0.916421 & 0.909631 \\
1.00 & 1.0 & 1.0 & 1.0 & 0.804257 & 0.999023 & 1.0 \\
\hline
\end{tabular}

\section{Comparison}

Modeled partial differentials equations with transformed boundary conditions were already solved analytically using the HPM, HAM, DTM, MDTM, and numerically using the fourthorder RungeKutta method by Keimanesh et al. in [28]. We proposed quadratic Lagrange Galerkin finite element method, thereby incorporating effectively the non-linearity of the flow problem. In order to verify the efficiency of the proposed method, a comparison between HPM, HAM, DTM, MDTM, NUM (numerical solutions using fourth-order RungeKutta method), and FEM (using quadratic Lagrange Galerkin finite element method) for various parameters is presented. Table 1 shows the comparison of results obtained for plane Poiseuille flow, while Table 2 is showing comparison of results obtained for generalized Couette flow. It is obvious that HPM is less accurate and is valid only for weak nonlinearity, the MDTM is a reliable algorithm method for solving highly nonlinear differential equations. It is obvious from the comparison tables that our proposed scheme is well behaved, convergent, and efficient. The accuracy of our proposed method is excellent in comparison with HPM, HAM, DTM, MDTM, NUM (numerical solutions using fourth-order RungeKutta method.)

\section{Conclusion}

The heat transfer phenomenon of a steady incompressible third grade flow in a channel subject to assumptions of no temperature jump and no slip at boundary is discussed. Numerical solution using a quadratic Lagrange Galerkin finite element method is proposed, thereby incorporating effectively the non-linearity of the flow problem. Three flow configurations, Couette, Poiseuille and plane Couette-Poiseuille, have been discussed. Finally, it has been substantiated that the fluid rheology and heat transfer phenomenon are greatly influenced by the third grade flow parameters, Brinkman number and pressure gradient using numerical simulations. Since, no à priori assumptions are made on pertinent flow parameters, apart from those due to thermodynamic stability, the results presented in this investigation are also valid for their large values. It is also noted that the fluid velocity depends upon the non-Newtonian parameter $\beta$ except in the case of simple plane Couette flow where it does not depend on this parameter. Moreover, 
the velocity increases with increasing value of $\beta$ and/or $B$. In case of plane Couette-Poiseuille flow a tendency of back flow is observed at the lower plate when $\beta$ and $B$ are greater than a specific value. This tendency of back flow becomes more and more dominating when values of $\beta$ and $B$ are increased beyond this value. In all the three cases the fluid temperature depends upon $\beta, B, k$, and it rises very rapidly with increasing values of these parameters.

\section{A Element Matrices for $P_{2}$}

Recall that the $P_{2}$-Lagrange interpolation functions $N_{j}^{i}$ for $j=1,2,3$ have the following expressions

$$
\left\{\begin{array}{l}
N_{1}^{i}(y)=1-3\left(\frac{y-y_{i}}{h}\right)+2\left(\frac{y-y_{i}}{h}\right)^{2}, \\
N_{2}^{i}(y)=1-4\left(\frac{y-y_{i+1 / 2}}{h}\right)^{2}, \\
N_{3}^{i}(y)=1-3\left(\frac{y_{i+1}-y}{h}\right)+2\left(\frac{y_{i+1}-y}{h}\right)^{2} .
\end{array}\right.
$$

Therefore, the element assembly matrices $\mathbf{A}_{j}^{i}$, for $j=1,2$, can be given by

$$
\mathbf{A}_{j}^{i}=\left(\begin{array}{ccc}
-\frac{7}{3} h & \frac{8}{3} h & -\frac{1}{3} h \\
\frac{8}{3} h & -\frac{16}{3} h & \frac{8}{3} h \\
-\frac{1}{3} h & \frac{8}{3} h & -\frac{7}{3} h
\end{array}\right)
$$

Similarly, we evaluate the components of the non-linear load matrices $\mathcal{N}_{j}^{i} \in \mathbb{R}^{3}$ for $j=1,2$. Let $\mathcal{N}_{j, k}^{i}$, for $k=1,2,3$, denote the $k^{t h}$ component of the vector $\mathcal{N}_{j}^{i}$. Then, we have

$$
\begin{aligned}
& \mathcal{N}_{1,1}^{i}=\frac{4 \beta}{5 h^{3}}\left(37\left(w_{1}^{i}\right)^{3}\right.+51\left(w_{1}^{i}\right)^{2} w_{3}^{i}-162\left(w_{1}^{i}\right)^{2} w_{2}^{i}+11 w_{1}^{i}\left(w_{3}^{i}\right)^{2}-124 w_{1}^{i} w_{2}^{i} w_{3}^{i} \\
&\left.+224 w_{1}^{i}\left(w_{2}^{i}\right)^{2}-3\left(w_{3}^{i}\right)^{3}-2 w_{2}^{i}\left(w_{3}^{i}\right)^{2}+64 w_{3}^{i}\left(w_{2}^{i}\right)^{2}-96\left(w_{2}^{i}\right)^{3}\right) \\
& \mathcal{N}_{1,2}^{i}=\frac{16 \beta}{5 h^{3}}\left(9\left(w_{1}^{i}\right)^{3}+\right. 7\left(w_{1}^{i}\right)^{2} w_{3}^{i}-34\left(w_{1}^{i}\right)^{2} w_{2}^{i}+7 w_{1}^{i}\left(w_{3}^{i}\right)^{2}-28 w_{1}^{i} w_{2}^{i} w_{3}^{i} \\
&\left.+48 w_{1}^{i}\left(w_{2}^{i}\right)^{2}+9\left(w_{3}^{i}\right)^{3}-34 w_{2}^{i}\left(w_{3}^{i}\right)^{2}+48 w_{3}^{i}\left(w_{2}^{i}\right)^{2}-32\left(w_{2}^{i}\right)^{3}\right) \\
& \mathcal{N}_{1,3}^{i}=\frac{-4 \beta}{5 h^{3}}\left(3\left(w_{1}^{i}\right)^{3}-\right. 11\left(w_{1}^{i}\right)^{2} w_{3}^{i}+2\left(w_{1}^{i}\right)^{2} w_{2}^{i}-51 w_{1}^{i}\left(w_{3}^{i}\right)^{2}+124 w_{1}^{i} w_{2}^{i} w_{3}^{i} \\
&\left.-64 w_{1}^{i}\left(w_{2}^{i}\right)^{2}-37\left(w_{3}^{i}\right)^{3}+162 w_{2}^{i}\left(w_{3}^{i}\right)^{2}-224 w_{3}^{i}\left(w_{2}^{i}\right)^{2}+96\left(w_{2}^{i}\right)^{3}\right),
\end{aligned}
$$


and

$$
\begin{aligned}
& \mathcal{N}_{2,1}^{i}=\frac{\lambda}{210 h^{3}}\left(31680 \beta\left(\gamma_{1}^{i}\right)^{2}\left(\gamma_{2}^{i}\right)^{2}-576 \beta\left(\gamma_{2}^{i}\right)^{2}\left(\gamma_{3}^{i}\right)^{2}+259\left(\gamma_{1}^{i}\right)^{2} h^{2}+7680 \beta\left(\gamma_{2}^{i}\right)^{4}\right. \\
& -12192 \beta\left(\gamma_{1}^{i}\right)^{2} \gamma_{2}^{i} \gamma_{3}^{i}+14976 \beta \gamma_{1}^{i}\left(\gamma_{2}^{i}\right)^{2} \gamma_{3}^{i}-2784 \beta \gamma_{1}^{i} \gamma_{2}^{i}\left(\gamma_{3}^{i}\right)^{2} \\
& -616 h^{2} \gamma_{1}^{i} \gamma_{2}^{i}+98 h^{2} \gamma_{1}^{i} \gamma_{3}^{i}-56 h^{2} \gamma_{2}^{i} \gamma_{3}^{i}-26112 \beta \gamma_{1}^{i}\left(\gamma_{2}^{i}\right)^{3} \\
& -17056 \beta\left(\gamma_{1}^{i}\right)^{3} \gamma_{2}^{i}+72 \beta \gamma_{1}^{i}\left(\gamma_{3}^{i}\right)^{3}+1284 \beta\left(\gamma_{1}^{i}\right)^{2}\left(\gamma_{3}^{i}\right)^{2}+1312 \beta \gamma_{2}^{i}\left(\gamma_{3}^{i}\right)^{3} \\
& -4608 \beta\left(\gamma_{2}^{i}\right)^{3} \gamma_{3}^{i}-346 \beta\left(\gamma_{3}^{i}\right)^{4}+3462 \beta\left(\gamma_{1}^{i}\right)^{4}+3208 \beta\left(\gamma_{1}^{i}\right)^{3} \gamma_{3}^{i} \\
& \left.+336 h^{2}\left(\gamma_{2}^{i}\right)^{2}-21 h^{2}\left(\gamma_{3}^{i}\right)^{2}\right) \text {, } \\
& \mathcal{N}_{2,2}^{i}=\frac{2 \lambda}{105 h^{3}}\left(3648 \beta\left(\gamma_{1}^{i}\right)^{2}\left(\gamma_{2}^{i}\right)^{2}+3648 \beta\left(\gamma_{2}^{i}\right)^{2}\left(\gamma_{3}^{i}\right)^{2}+63\left(\gamma_{1}^{i}\right)^{2} h^{2}+1536 \beta\left(\gamma_{2}^{i}\right)^{4}\right) \\
& -960 \beta\left(\gamma_{1}^{i}\right)^{2} \gamma_{2}^{i} \gamma_{3}^{i}+1920 \beta \gamma_{1}^{i}\left(\gamma_{2}^{i}\right)^{2} \gamma_{3}^{i}-960 \beta \gamma_{1}^{i} \gamma_{2}^{i}\left(\gamma_{3}^{i}\right)^{2}-112 h^{2} \gamma_{1}^{i} \gamma_{2}^{i} \\
& -14 h^{2} \gamma_{1}^{i} \gamma_{3}^{i}-112 h^{2} \gamma_{2}^{i} \gamma_{3}^{i}-3072 \beta \gamma_{1}^{i}\left(\gamma_{2}^{i}\right)^{3}-2112 \beta\left(\gamma_{1}^{i}\right)^{3} \gamma_{2}^{i} \\
& +104 \beta \gamma_{1}^{i}\left(\gamma_{3}^{i}\right)^{3}+324 \beta\left(\gamma_{1}^{i}\right)^{2}\left(\gamma_{3}^{i}\right)^{2}-2112 \beta \gamma_{2}^{i}\left(\gamma_{3}^{i}\right)^{3}-3072 \beta\left(\gamma_{2}^{i}\right)^{3} \gamma_{3}^{i}\left(\gamma_{3}^{i}\right)^{4} \\
& \left.+502 \beta+502 \beta\left(\gamma_{1}^{i}\right)^{4}+104 \beta\left(\gamma_{1}^{i}\right)^{3} \gamma_{3}^{i}+112 h^{2}\left(\gamma_{2}^{i}\right)^{2}+63 h^{2}\left(\gamma_{3}^{i}\right)^{2}\right) \text {, } \\
& \mathcal{N}_{2,3}^{i}=-\frac{\lambda}{210 h^{3}}\left(576 \beta\left(\gamma_{1}^{i}\right)^{2}\left(\gamma_{2}^{i}\right)^{2}-72 \beta\left(\gamma_{1}^{i}\right)^{3} \gamma_{3}^{i}+21 h^{2}\left(\gamma_{1}^{i}\right)^{2}-7680 \beta\left(\gamma_{2}^{i}\right)^{4}\right. \\
& +2784 \beta\left(\gamma_{1}^{i}\right)^{2} \gamma_{2}^{i} \gamma_{3}^{i}-14976 \beta \gamma_{1}^{i}\left(\gamma_{2}^{i}\right)^{2} \gamma_{3}^{i}+12192 \beta \gamma_{1}^{i} \gamma_{2}^{i}\left(\gamma_{3}^{i}\right)^{2}+56 h^{2} \gamma_{1}^{i} \gamma_{2}^{i} \\
& -98 h^{2} \gamma_{1}^{i} \gamma_{3}^{i}+616 h^{2} \gamma_{2}^{i} \gamma_{3}^{i}-3208 \beta \gamma_{1}^{i}\left(\gamma_{3}^{i}\right)^{3}-1312 \beta\left(\gamma_{1}^{i}\right)^{3} \gamma_{2}^{i}+4608 \beta \gamma_{1}^{i}\left(\gamma_{2}^{i}\right)^{3} \\
& -1284 \beta\left(\gamma_{1}^{i}\right)^{2}\left(\gamma_{3}^{i}\right)^{2}+17056 \beta \gamma_{2}^{i}\left(\gamma_{3}^{i}\right)^{3}+26112 \beta\left(\gamma_{2}^{i}\right)^{3} \gamma_{3}^{i}-3462 \beta\left(\gamma_{3}^{i}\right)^{4} \\
& \left.+346 \beta\left(\gamma_{1}^{i}\right)^{4}-336 h^{2}\left(\gamma_{2}^{i}\right)^{2}-259 h^{2}\left(\gamma_{3}^{i}\right)^{2}-31680 \beta\left(\gamma_{2}^{i}\right)^{2}\left(\gamma_{3}^{i}\right)^{2}\right) .
\end{aligned}
$$

Finally, denoting the $k^{t h}$ component of the vector $\mathbf{B}_{j}^{i}$ by $B_{j, k}^{i}$, we find out that

$$
B_{j, 1}^{i}=-\frac{C_{j} h}{6}, \quad B_{j, 2}^{i}=-\frac{2 C_{j} h}{3} \quad \text { and } \quad B_{j, 3}^{i}=-\frac{C_{j} h}{6} .
$$

\section{References}

[1] J. G. Oldroyd, On the formulation of rheological equations of state, Proceedings of the Royal Society of London: Series A, 200(5): (1950), pp. 23-41.

[2] R. B. Bird, R. C. Armstrong, and O. Hassager, Dynamics of polymeric liquids in Fluid mechanics (Vol. 1 ), Wiley-Interscience, New York, 1987.

[3] A. M. Siddiqui, M. Hameed, B. M. Siddiqui, and Q. K. Ghori, Use of Adomian decomposition method in the study of parallel plate flow of a third grade fluid, Communications in Nonlinear Science and Numerical Simulation, 15(9): (2010), pp. 2388-2399.

[4] M. Danish, S. Kumar, and S. Kumar, Exact analytical solutions for the Poiseuille and Couette-Poiseuille flow of third grade fluid between parallel plates, Communications in Nonlinear Science and Numerical Simulation, 17: (2012), pp. 1089-1097. 
[5] M. Hatamia, J. Hatamic, and D. D. Ganji, Computer simulation of MHD blood conveying gold nanoparticles as a third grade non-Newtonian nanofluid in a hollow porous vessel, Computer Methods and Programs in Biomedicine, 113(2): (2014), pp. 632-641.

[6] M. Hosseini, Z. Sheikholeslami, and D. D. Ganji, Non-Newtonian fluid flow in an axisymmetric channel with porous wall, Propulsion and Power Research, 2(4):(2013), pp. 254-262.

[7] J. Rahimi, D. D. Ganji, M. Khaki, and Kh. Hosseinzadeh, Solution of the boundary layer flow of an Eyring-Powell non-Newtonian fluid over a linear stretching sheet by collocation method, Alexandria Engineering Journal, doi.org/10.1016/j.aej.2016.11.006, In Press.

[8] H. R. Ashorynejad, K. Javaherdeh, M. Sheikholeslami, and D. D. Ganji, Investigation of the heat transfer of a non-Newtonian fluid flow in an axisymmetric channel with porous wall using Parameterized Perturbation Method (PPM), Journal of the Franklin Institute, 351(2): (2014), pp. 701-712.

[9] M. Rahimi-Gorji, O. Pourmehran , M. Gorji-Bandpy, and D. D. Ganji, An analytical investigation on unsteady motion of vertically falling spherical particles in non-Newtonian fluid by Collocation Method, Ain Shams Engineering Journal, 6(2): (2015), pp. 531-540.

[10] M. Hatami, and D. D. Ganji, Natural convection of sodium alginate (SA) non-Newtonian nanofluid flow between two vertical flat plates by analytical and numerical methods, Case Studies in Thermal Engineering, 2: (2014), pp. 14-22.

[11] R. P. Chhabra, and J. F. Richardson, Non-Newtonian Flow and Applied Rheology (2nd ed.), Butterworth-Heinemann, Oxford, 2008.

[12] J. M. Ekmann, D. J. Wildman, and J. L. S. Chen, Laminar flow studies of highly loaded suspensions in horizontal pipes. Second Intl. Symp. Slurry Flows ASME FED 38 (85): (1986).

[13] S. M. Richardson, Flows of variable-viscosity fluids in ducts with heated walls. J. NonNewtonian Fluid Mech. 25: (1987), pp. 137-156.

[14] M. Massoudi and T. X. Phuoc, Flow of a generalized second grade non-Newtonian fluid with variable viscosity, Continuum Mech. Thermodyn. 16:(2004), pp. 529-538.

[15] O. Aydin and M. Avci, Laminar Forced Convection with Viscous Dissipation in a CouettePoiseuille Flow between Parallel Plates, Applied Energy, 83(8): (2006), pp. 856-867.

[16] Y. L. Chen and K. Q. Zhu, Couette-Poiseuille Flow of Bingham Fluids between Two Porous Parallel Plates with Slip Conditions, Journal of Non-Newtonian Fluid Mechanics, 153(1): (2008), pp. 1-11.

[17] Y. Liu and K. Zhu, Study of shear-thinning/thickening effects on plane Couette-Poiseuille flow with uniform crossflow, Appl. Math. Mech. -Engl. Ed., 35(5): (2014), 549-566.

[18] M. Imran, M. Tahir, M. Nazar, and M. Kamran, Some Couette flows of a second grade fluid due to tangential stresses, Science International, 27(3): (2015), pp. 1755-1760.

[19] L. Klotz, G. Lemoult, I. Frontczak, L. S. Tuckerman, and J. E. Wesfreid, Couette-Poiseuille flow experiment with zero mean advection velocity: Subcritical transition to turbulence, Physical Review Fluids, 2: (2017), 043904. 
[20] R. Mahmood, M. Sajid, and A. Nadeem, Finite element solution for heat transfer flow of a third order fluid between parallel plates, Adv. Studies Theor. Phys., 5(3):2011, pp. $107-120$.

[21] A. M. Siddiqui, A. Zeb, Q. K. Ghori and A. M. Benharbit, Homotopy perturbation method for heat transfer flow of a third grade fluid between parallel plates, Chaos, Solitons and Fractals, 36: (2008), pp. 182-192.

[22] A. Rasheed, R. Nawaz, S. A. Khan, Hanifa, and A. Wahab, Numerical study of a thin film flow of fourth grade fluid, International Journal of Numerical Methods for Heat and Fluid Flow, 25(4): (2015), pp. 929-940.

[23] A. Wahab, A. Rasheed, R. Nawaz, and N. Javaid, Numerical study of two dimensional unsteady flow of an anomalous Maxwell fluid, International Journal of Numerical Methods for Heat and Fluid Flow, 25(5): (2015), pp. 1120-1137.

[24] T. Hayat, S. Qayyum, M. Farooq, A. Alsaedi, and M. Ayub, Mixed convection flow of Jeffrey fluid along an inclined stretching cylinder with double stratification effect, Thermal Science, doi:10.2298/TSCI141106052H.

[25] M. Sajid, T. Hayat, and S. Asghar, Comparison of the HAM and HPM solutions of thin film flows of non-Newtonian fluids on a moving belt, Nonlinear Dynamics, 50: (2007), pp. $27-35$.

[26] H. Brezis, Functional Analysis, Sobolev Spaces and Partial Differential Equations, Springer-Verlag, 2011.

[27] P. G. Ciarlet, The Finite Element for Elliptic Problems, Classics in Applied Mathematics, SIAM, USA, 1979.

[28] M. Keimanesh, M. M. Rashidi, A. J. Chamkha, and R. Jafari, Study of a third grade nonNewtonian fluid flow between two parallel plates using the multi-step differential transform method, Computers and Mathematics with Applications, 62: (2011), pp. 2871-2891. 